\title{
A biomimetic sliding-stretching approach to seismic isolation
}

\author{
Fernando Fraternali $[$ Narinder Singh $(\mathbb{D} \cdot$ \\ Ada Amendola $\mathbb{D}$ - Gianmario Benzoni • \\ Graeme W. Milton(D)
}

Received: 4 July 2021 / Accepted: 9 October 2021 / Published online: 2 November 2021

(C) The Author(s) 2021

\begin{abstract}
There is growing demand in industrialized and developing countries to provide people and structures with effective earthquake protection. Here, we employ architectured material concepts and a bioinspired approach to trail-blaze a new path to seismic isolation. We develop a novel seismic isolator whose unit cell is formed by linkages that replicate the bones of human limbs. Deformable tendons connect the limb members to a central post carrying the vertical load, which can slide against the bottom plate of the system. While the displacement capacity of the device depends only on the geometry of the limbs, its vibration period is tuned by dynamically stretching the tendons in the nonlinear stress-strain regime, so as to avoid resonance with seismic excitations. This biomimetic, sliding-stretching isolator can be scaled to seismically protect infrastructure, buildings, artworks and equipment with customized properties and sustainable materials. It does not require heavy industry or expensive materials and is easily assembled from metallic parts and 3D-printed components.
\end{abstract}

Supplementary Information The online version contains supplementary material available at https://doi.org/10.1007/ s11071-021-06980-5.

F. Fraternali $(\varangle) \cdot$ N. Singh · A. Amendola · G. Benzoni Department of Civil Engineering, University of Salerno, 84084 Fisciano, SA, Italy

e-mail: f.fraternali@unisa.it

G. W. Milton

Department of Mathematics, University of Utah, Salt Lake City, UT 84112, USA
Keywords Seismic isolation Architectured materials - Nonlinear behavior - Sliding - Stretching . Biomimetic response $\cdot 3 \mathrm{D}$ printing

\section{Introduction}

In the event of earthquakes, seismic isolation offers an effective strategy to ensure the safety of people and the prevention of damage to structures, machinery and equipment [1-6]. The most widespread seismic isolators currently on the market use elastomeric or friction-pendulum bearings [7,8]. These devices partially or completely disconnect the portion of the 'superstructure' above them from the ground motion. As a result, the fundamental vibration period of the structure is significantly increased, avoiding resonance with high-frequency content seismic excitations [9]. Seismic isolators offer different levels of damping [10], which is useful for dissipating energy and reducing the amplitude of lateral displacements during earthquakes. The inherent limitations of currently available isolators include their confined operational frequencies, manufacturing complexity, need for advanced technical expertise and substantial costs.

While elastomeric bearings are still widely used and appreciated for their simplicity and consistent performance, even after several decades in service [11-13], the friction-based devices become quickly the technology of choice when the displacement demand increases significantly. Sliding bearings generally imply a more 
complex behavior of the isolated structure, due to the intrinsic complexity of the frictional phenomena and the still limited data about the durability of their critical components [14]. The choice of the appropriate technology for seismic isolation interventions on existing or new structures requires a careful analysis $[1,15]$. However, even the simplest implementation of the seismic isolation technology offers a major improvement in terms of level of seismic protection of buildings and infrastructures in both industrialized and developing countries. Residential and rural buildings [16], equipment in hospitals and essential buildings [4,5], artworks in museums [6] and critical industrial facilities are nowadays often protected through many variations and combinations of seismic isolators. The considerable high cost and the perceived complexity of such devices, together with local availability constraints, often discourage a larger use of this reliable technology, particularly in less developed countries. The scientific community senses the need to create the conditions for a broader field of applications, and in this sense several low-cost solutions for seismic isolators have been proposed in the literature and are currently under investigation $[4,16-19]$.

The use of architectured material concepts to design next-generation seismic isolators deserves special attention, in consideration of the exceptional properties these systems exhibit in different fields of mechanics, robotics and acoustics [21-27]. This is due to an optimized geometric design of the internal structure $[20,21]$ that can be conveniently manufactured using 3D printing at different scales [28-30]. Seismic metamaterials have been successfully proposed to protect buildings from seismic waves by creating shields around the structure through Bragg-scattering structured soils, buried mass or above-surface resonators, auxetic and hierarchical materials [31-36]. The periodicity of these media is often at the meter scale, which makes them not suited for use as seismic isolators. The employment of confined pentamode lattices as smallamplitude vibration attenuation devices has been the subject of recent discussion in the literature [37].

Ever since Leonardo da Vinci's pioneering anatomic studies [38], the mechanics of human and animal locomotion has attracted researchers' attention. Animals adjust their muscle contraction frequencies to reach a state of resonance with pendulum- and elastictype oscillation mechanisms during locomotion [39]. This frequency tuning process produces motion at low energy consumption. The bones of the legs and arms behave as pendulum systems that the muscles bend periodically so as to match their natural frequencies [40,41]. Animals such as jellyfish tune vibration frequencies through the elasticity of their tissues, which is a nonlinear function of the deflection of the mesoglea bell [39].

This work takes a novel approach to a bio-inspired design of seismic isolation systems. We design a hybrid sliding-stretching isolator (SSI) combining finite kinematics linkages that replicate the bones of human arms and legs, stretchable membranes that mimic the action of the muscle-tendon complex and confinement plates. The proposed device dissipates mechanical energy via friction and the hysteretic recentering force of the tendons. Its displacement capacity can be finely tuned through an optimized design of the geometry of the limb members, while dissipative effects can be adjusted for the application at hand by playing with the geometry, the training cycles and the material of the tendons. The SSI can be manufactured on-site or in a fabrication laboratory using $3 \mathrm{D}$ printers and metallic parts provided by local metal framing companies or online suppliers, and hence does not require heavy industry or expensive materials.

\section{Materials and methods}

\subsection{Unit cell design}

An exploded view of the unit cell of the SSI is shown in Fig. 1. It is composed of four articulated 'limb' members (arms and legs) branching out from a central post connected to the top plate of the device. This plate carries the vertical load transmitted by the superstructure, consisting, e.g., of a column, a bridge beam, machinery or an artifact that needs to be isolated from the foundation. A cap screwed to the top plate covers the central post, which cap can exhibit relative rotations with respect to the post, due to a deformable cap cushion positioned between these parts. Such a movement allows the top plate to achieve the correct level (Fig. 1). If rocking motions need to be prevented [5], the cushion can be removed, with the cap and the post forming a single element. The central post ends with a slider made of a low-friction material built into a recess in its base. As Fig. 1 illustrates, four corner posts are screwed to the bottom plate and connected to the limb members at 


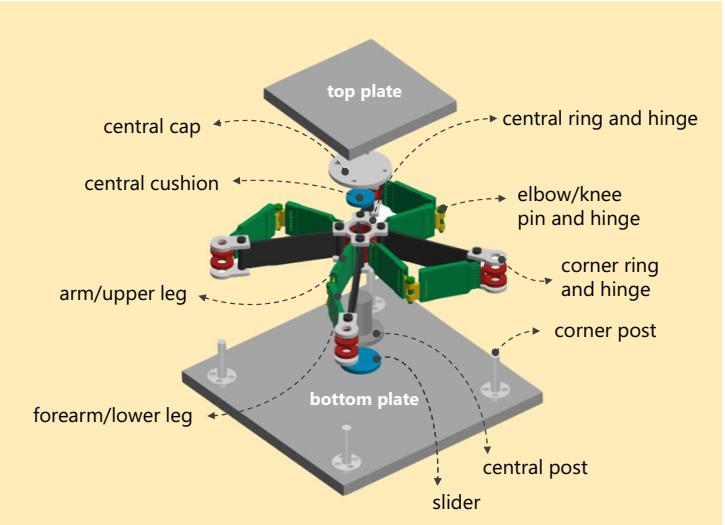

Fig. 1 Exploded view of the SSI unit cell

the ends opposite the central post. Vertical hinges connect the limbs to one another and to the posts, while four membranes-tendons connect the corner posts to the central post (see Fig. S1 and the mounting tutorial provided in Movie S1). The tendons are made of a material that can carry large stretching strains and that dissipates energy through hysteretic response [43,44]. The undeformed configuration of the limbs, depicted with red dashed lines in Fig. 2a, shows lower arms and legs with axial length $a_{1}$, upper arms and legs with axial length $a_{2}$, and the relative angle between these members equal to $\pi / 2+\beta$. Interestingly, the design variable $\beta$ ('initial foot angle' or 'rest angle' of the limbs) can be easily varied by changing the screw points of the corner posts on the bottom plate.

The kinematics of the unit cell is described by the two parameters illustrated in Fig. 2a, for a given direction of the displacement of the central node (relative to the foundation): the angle $\alpha$ formed by such a direction with the horizontal axis, and the scalar projection $u$ of the displacement vector of the central node in this direction. The displaced positions of the elbow and knee joints are easily found at the intersection points of the circle with radius $a_{2}$, which is centered at the current position of the central post, and the circles with radius $a_{1}$ centered at the corner posts. Due to symmetry, the kinematics of the cell is comprehensively studied by assuming $u>0$ and letting $\alpha$ vary in the interval $[0,90]$ deg (see Supplementary Text for analytic results). It is safe to assume that the design value of the lateral displacement allowed by the biomimetic isolator, hereafter referred to as 'displacement capacity' $d$, must be sufficiently lower than the minimum value of $u$ producing a 'locking' configuration of the unit cell $\left(u=u_{\text {lock }}\right)$. The locking condition is achieved when one or more tendons overlap the adjacent limb members (see Fig. 2b; Fig. S4 and Movie S2).

The plot in Fig. $2 \mathrm{~b}$ shows the variation of the dimensionless locking displacement $\bar{u}_{\text {lock }}=u_{\text {lock }} / a$ with $\alpha$, for $a_{1}=a_{2}=a$. One observes that the minimum value of $\bar{u}_{\text {lock }}$ is attained at $\alpha=\pi / 4-\beta / 2$, and that such a value grows appreciably when the rest angle changes from positive to negative. Indeed, it increases from 0.468 to $0.714(\approx 53 \%)$ when switching $\beta$ from $10 \mathrm{deg}$ to $-10 \mathrm{deg}$. It is also possible to double the displacement capacity of the device by stacking 2 unit cells one over the other in the vertical direction, as shown in Fig. 2c (see also Fig. S2, D). The remarkable increase in $d$ in systems with negative rest angles is achieved without changing the limbs' length and actually slightly reducing the footprint of the device (see insets in Fig. $2 b)$. Overall, the displacement capacity depends only on the geometric design variables $a_{1}, a_{2}$ and $\beta$ (see also Movie S2).

Positive values of the rest angle are needed to build periodic systems formed by arrays of unit cells, since the knee/elbow joints of adjacent cells do not touch one another if $\beta$ is sufficiently greater than zero (Fig. S2, B). This design approach distributes the vertical load $P$ transmitted by the superstructure among multiple central posts, creating a periodic metamaterial.

The bio-inspired character of the proposed seismic isolator is multifold. First, the shape of the unit cell replicates that of a human body with bent arms and legs (Figs. 1, 2 and 3). Second, the tendon-membranes' capacity to stretch acts as a recentering mechanism of the central post, which regulates the fundamental vibration period of the isolated structure. The extension-andrelease working principle of the membranes produces time fluctuations of kinetic and stored energies similar to those induced by the stretching and recoil of animals' tendons during running and hopping [40]. Such a response also converts the frequency tuning mechanism associated with the locomotion of animals [39] into a passive mode. While animals move at resonance through the active control of locomotion by muscles, tendons and tissues, the SSI analyzed in this study works in an opposite fashion: it tunes the nonlinear stiffness of the tendons to avoid resonance with seismic excitation frequencies [9]. It is also possible to actively control the isolator by equipping the device with actuated cables that run on top of the posts or along the 
A

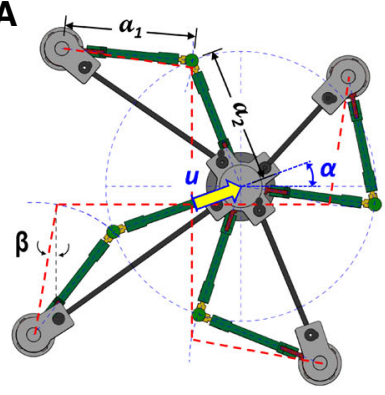

C

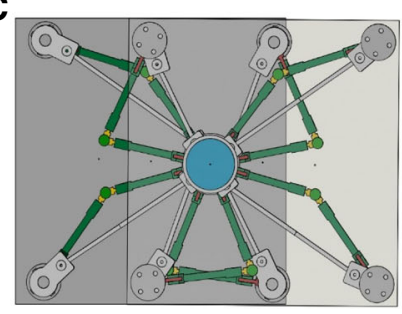

B
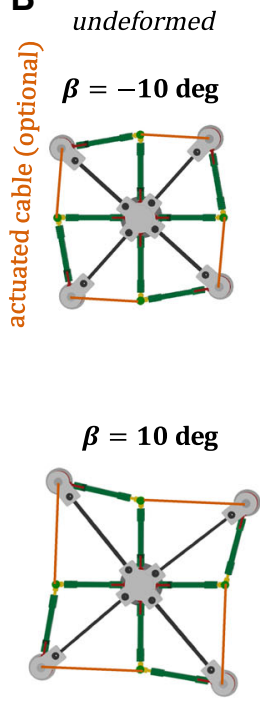

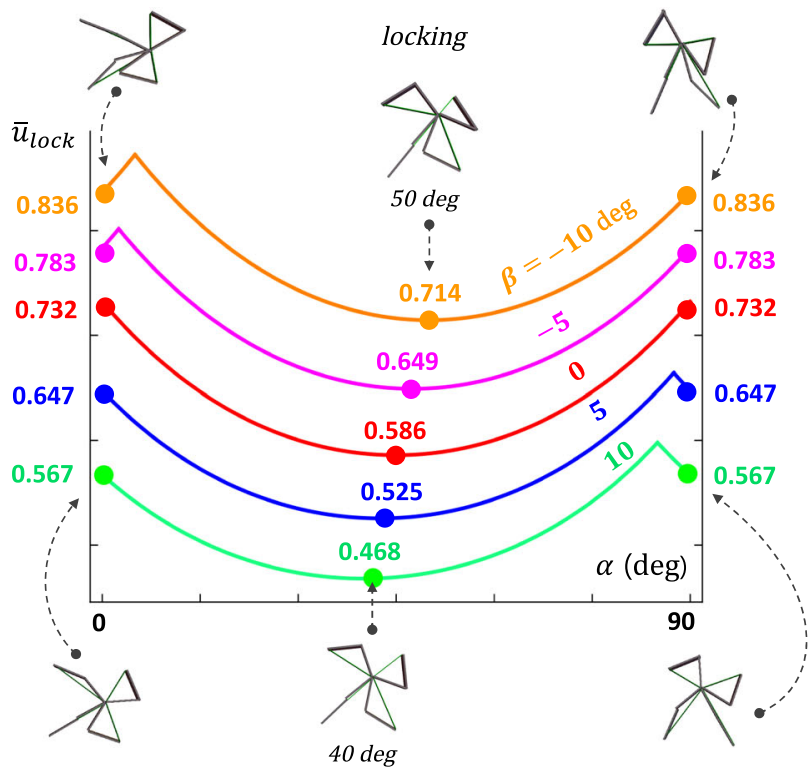

Fig. 2 Kinematic response. a Kinematics of the unit cell. b Locking displacements versus loading angle $\alpha$ and rest angle $\beta$. c Deformed configuration of a two-layer system (see also Fig. S2, D)

tendons controlled, e.g., by actuators embedded in the joints [42] (Fig. 2b and Fig. S2, B). This optional feature can be useful because it allows adjustment in real time of the fundamental vibration period and the dissipation capacity of the isolated structure to the earthquake frequency and energy content during extreme events [3]. The active control provides an additional recentering mechanism of the system.

\subsection{Preparation of samples}

Physical models of the SSI were manufactured at the Rapid Prototyping Laboratory of the University of Salerno. This was achieved using fused deposition modeling (FDM) 3D printers, a lathe from a partner metal framing company and Aluminum 7075-T651 (Ergal) confinement plates (see Supplementary Materials for details). The analyzed prototypes feature a single unit cell with the properties $a_{1}=97.0 \mathrm{~mm}$, $a_{2}=100.5 \mathrm{~mm}, \beta=0$ and overall height of $95 \mathrm{~mm}$ (including the confinement plates). The non-structural components were 3D printed using eco-friendly polylactic acid (PLA) filaments. The load-carrying members of the tested prototypes were fabricated in S235 steel through a parallel lathe (Fig. S1). The slider underneath the central post (Fig. 1) is a circular disk made of polytetrafluoroethylene (PTFE). One of the tested prototypes (prototype \#1) does not have tendons and was analyzed to study the pure-friction sliding response of the central post (Fig. 3a). Prototype \#2 features tendons 3D-printed using a thermoplastic polyurethane (TPU) filament for FDM (Fig. 3d and Movie S3). Prototypes equipped with fully 3D-printed unit cell parts were also manufactured for demonstrative purposes (Fig. 3a,b and Movie S3). Table S1 of Supplementary Text illustrates the key manufacturing parameters of the biomimetic isolator prototypes.

\subsection{Experimental validation procedure}

Experimental validation tests were designed 'ad hoc' in collaboration with FIP MEC srl, a leading company in the field of antiseismic devices based in Padova (Italy). A loading frame equipped with vertical and horizontal hydraulic actuators (Fig. S3) was employed to apply unidirectional displacement histories to the bottom plate, while subjecting the top plate to a fixed vertical load $P$.

The testing activities led to the execution of 1 training cycle and 4 additional cycles of a sinusoidal displacement time-history with a frequency of $0.40 \mathrm{~Hz}$ and amplitude $d= \pm 50 \mathrm{~mm}$. Additional tests were run 

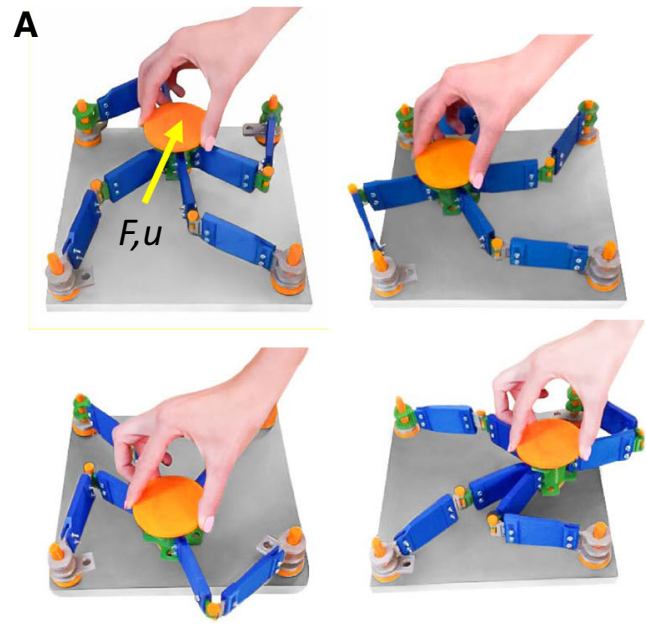

B

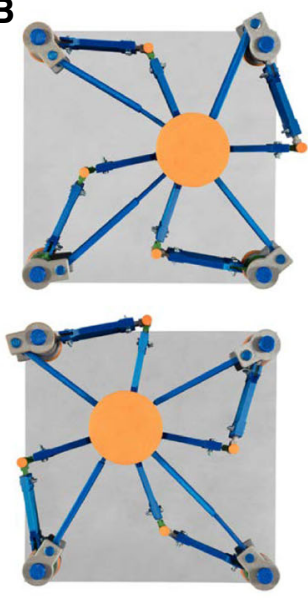

C
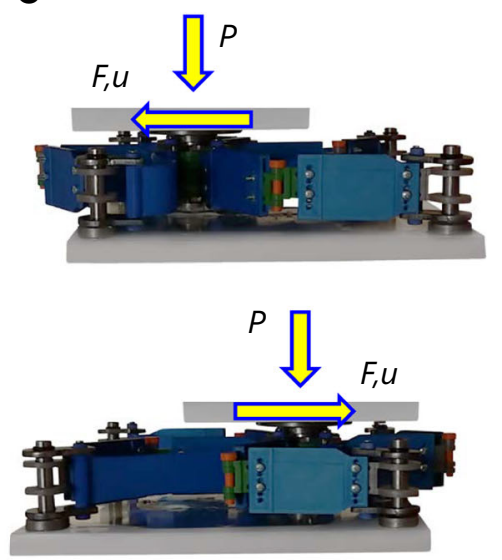

Fig. 3 Physical models and experimental validation. a Motion animation of a demonstrative version of prototype \#1 equipped with fully 3D-printed unit cell parts, which can be moved by hand. b Demonstrative version of prototype \#2 equipped with telescopic tendons (see Movie S3 for a motion video-clip). (c) Prototype \#2 with stretchable tendons and metallic posts under testing (a featured video is given in Movie S4)

sample \#2, after all the tests on this sample were completed. Preliminary mechanical characterization tests were run on the tendons, as detailed in Supplementary Text.

\section{Mechanical modeling}

\subsection{Nonlinear force-displacement response}

The cyclic uniaxial tension tests on the tendons presented in Supplementary Text are well described by the pseudo-elastic (PE) constitutive models by Dorfmann and Ogden for rubber-like materials [43,44]. Let $\lambda$ and $\hat{\sigma}_{t}$, respectively, denote the stretch ratio (after pre-conditioning) and the nominal stress carried by the tendons. Assuming a no-compression behavior (due to the thin-walled cross section), we model the tensile response of the tendons through the 'model 2' analytically described in Supplementary Text (PE model), at the strain rate of $0.11 / \mathrm{s}$ and up to $\approx 30 \%$ incremental strains from the pre-conditioned state. The maximum strain rate of $0.65 / \mathrm{s}$ observed in the tendons of sample \#2 under testing is significantly higher than that characterizing the cyclic tests on the tendons presented in Supplementary Materials. It has been reported in the literature that an increase in the strain rate may appreciably harden the stress-strain curve of TPU during 
the loading phase [45,46], while keeping the unloading branch essentially unchanged [45]. In order to account for high-strain-rate effects, we multiply the PE function $\hat{\sigma}_{t}(\lambda)$ by a scaling factor $(1+\psi)$, where $\psi$ denotes a strain rate parameter [47].

The overall restoring force $F_{r}$ transmitted from the tendons to the central post (Fig. S2, C) is computed through the equation:

$F_{r}=A_{t}\left(\sum_{j}(1+\psi) \hat{\sigma}_{t}\left(\lambda_{t, j}\right) \hat{\mathbf{k}}_{t, j}\right) \cdot \hat{\mathbf{k}}^{u}$

where now $A_{t}$ is the cross section area of the tendons in the rest configuration ; $\lambda_{t, j}$ and $\hat{\mathbf{k}}_{t, j}$ denote the stretch ratio and the unit vector associated with the tendon that links the $j$-th corner post with the central post ( $j=1,3,7,9$, see Fig. S2, A), respectively; and $\hat{\mathbf{k}}^{u}=\{\cos (\alpha), \sin (\alpha), 0\}$ indicates the unit vector in the direction of the applied displacement $u$. The no-compression assumption implies $\hat{\sigma}_{t}=0$ for any $\lambda_{t, j}<0$.

A second horizontal force $F_{f}$ acting on the central post follows from the friction resistance opposed by the slider-plate interface to the sliding movement (Fig. $\mathrm{S} 2, \mathrm{C})$. The constitutive law for $F_{f}$ can be obtained by generalizing the nonlinear friction model for concave sliding bearings by Lomiento et al. [8] to the SSI. Such a model assumes that the friction coefficient $\mu$ reduces its value with increasing values of the applied vertical load $P$ ('load' effect); varies with the sliding velocity $v=\dot{u}$ ('velocity' effect); slightly reduces under cycling loading ('cycling' effect); and sharply increases when the motion initiates or reverses its direction ('breakaway' effect). The experimental results presented in Fig. 4 highlight that the model by Lomiento et al. [8] can be reasonably applied to the SSI samples under examination by discarding the cycling and breakaway effects. We are therefore led to the following constitutive law for $F_{f}$ :

$F_{f}=\mu P \operatorname{sign}(v)$

where $v$ is the sliding velocity, $\operatorname{sign}(\cdot)$ denotes the signum function, and it results in:

$\mu=\mu_{s 0} e^{-\frac{P}{P_{r e f}}}\left(\gamma+(1-\gamma) e^{-\frac{|v|}{v_{r e f}}}\right)$.

Here, $\mu_{s 0}$ indicates the slow-motion coefficient of friction under zero vertical load; $P_{\text {ref }}$ and $v_{\text {ref }}$ denote reference values of the applied vertical load $P$ and sliding velocity, respectively; and $\gamma$ denotes a dimensionless scalar parameter greater than one [8].

\subsection{Effective dynamic properties}

We denote energy dissipated per cycle by the SSI through the symbol $E D C$. An effective value of the dynamic friction coefficient of prototype \#1 can be defined as follows:

$\mu_{e f f}=E D C /(4 P d)$.

Let now $F_{d}$ denote the value of the base shear force $F$ corresponding to the design displacement $u=d$ of prototype \#2. We compute the effective damping coefficient of this prototype through the following formula provided by international standards for seismic isolators [10]:

$\xi_{\text {eff }}=\frac{E D C}{2 \pi F_{d} d}$.

The effective period of vibration of the system is instead computed as follows:

$T_{\text {eff }}=2 \pi \sqrt{\frac{M}{F_{d} / d}}$.

$M=P / g$ being the mass of the superstructure (here $g$ denotes the gravitational acceleration). The above properties control both the extreme values of the seismic load suffered by the superstructure and the displacement drift between the superstructure and the foundation [48]. They are employed to characterize the fundamental performance target of the isolated building and to perform the selection of the appropriate isolation system in the pre-design phase [1]. The finetuning of the isolation system requires a detailed structural analysis of the complex formed by the superstructure and the seismic isolators, which accounts for the accurate modeling of the force-displacement response described in the previous section.

\section{Results and discussion}

Let $F=F_{r}+F_{s}$ denote the overall shear force acting on the top plate of the SSI (base shear of the superstructure). The experimental and theoretical results obtained for the shear force $F$ vs. lateral displacement $u$ response of prototype \#1 and \#2 are illustrated in Figs. 4 and 5, respectively, in correspondence with the examined values of the vertical load $(P=5,15,25 \mathrm{kN})$. A trial and error procedure was employed to obtain the best fit parameters of the friction model described by Eqs. 
A

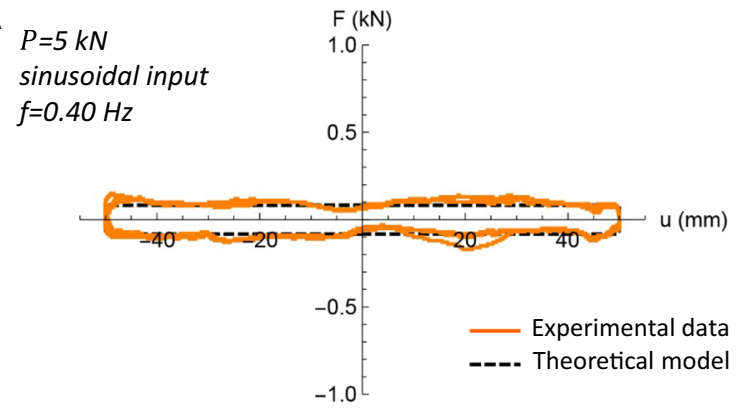

C

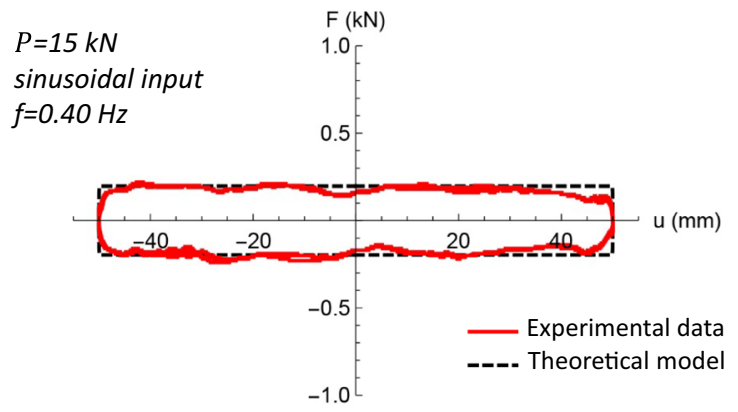

E

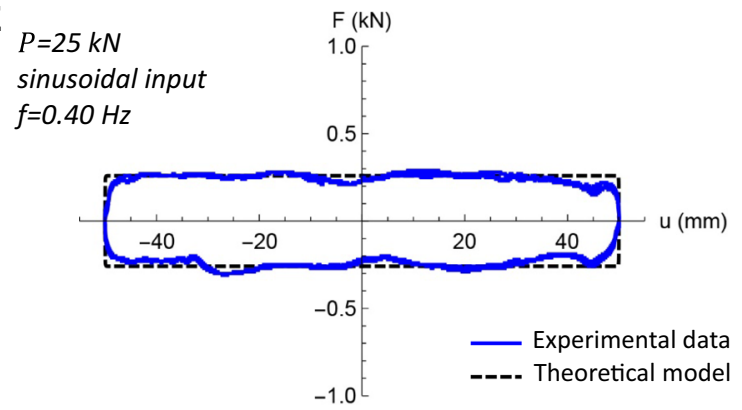

Fig. 4 Shear force $F$ versus lateral displacement $u$ response of the SSI prototype \#1. Comparison of experimental results and theoretical predictions under fixed vertical load $P$ and cyclic displacement histories with amplitude $d= \pm 50 \mathrm{~mm}$, for $P=5 \mathrm{kN}$ a,b); $P=15 \mathrm{kN} \mathrm{c,d}$; and $P=25 \mathrm{kN}(\mathbf{e , f})$. The displacement

(2-3) and the constitutive model given by Eq. (1) with respect to the experimental results for prototypes \#1 and \#2, respectively (after the training cycles). We estimated $P_{\text {ref }}=42.35 \mathrm{kN}, \mu_{s 0}=0.47 \%, \gamma=4.00$, $v_{\text {ref }}=2.50 \mathrm{~mm} / \mathrm{s}$ and $\psi=0.19$. The accuracy of the employed mechanical models for $F_{f}$ and $F_{r}$ is demonstrated by the good theory vs. experiment matching observed in Figs. 4 and 5.

For the tests run on prototype \#1, we note the following theory vs. experiment differences in terms of the
B

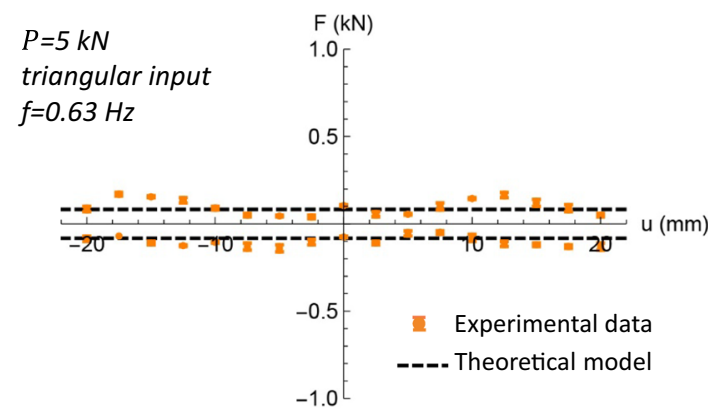

D

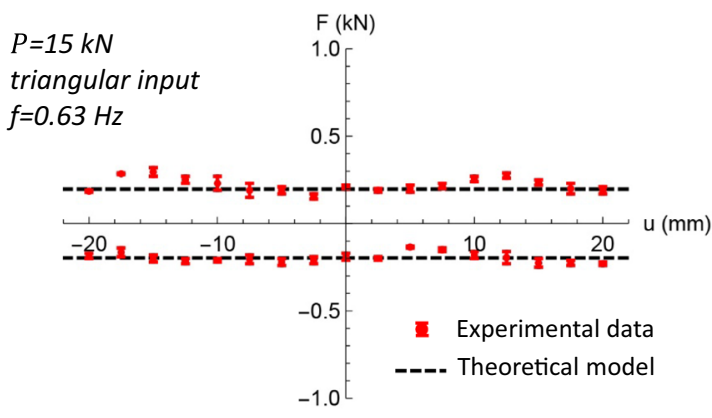

$\mathbf{F}$

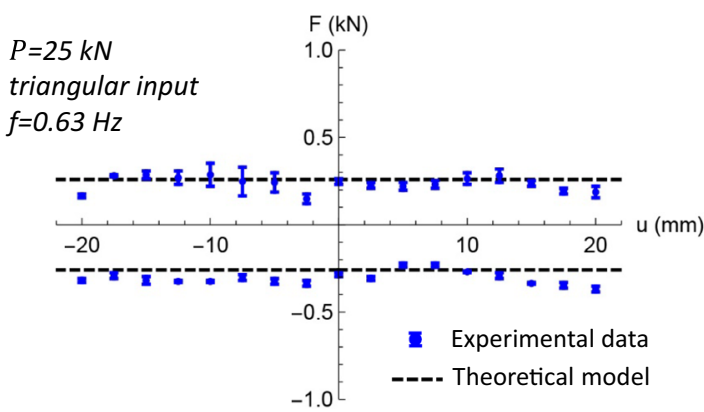

window of triangular loading tests has been restricted to $85 \%$ of the maximum value to exclude disturbance effects related to load reversal. Error bars refer to deviations of the shear force from the mean value under cyclic loading. Video recordings of selected tests are given in Movies S5-S7

average energy dissipated per cycle (sinusoidal tests): $-5.27 \%$ for $P=5 \mathrm{kN}$ (theory: $16.61 \mathrm{~J}$; experiment: $17.54 \mathrm{~J}) ; 11.07 \%$ for $P=15 \mathrm{kN}$ (theory: $39.36 \mathrm{~J}$; experiment: $35.44 \mathrm{~J}$ ); and $7.86 \%$ for $P=25 \mathrm{kN}$ (theory: 51.81 J; experiment: $48.03 \mathrm{~J})$. These values of $E D C$ correspond to average values of $\mu_{e f f}$ varying from $1.66 \%$ $(P=5 \mathrm{kN})$ to $1.04 \%(P=25 \mathrm{kN})$. The oscillations of the experimental results visible in Fig. 4 are explained by accuracy measurement errors due to the low values of the shear forces recorded during the tests $(0.1-0.3$ 
A

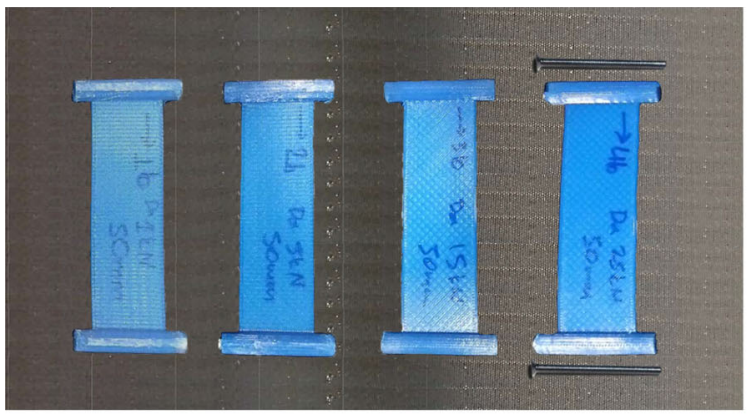

C

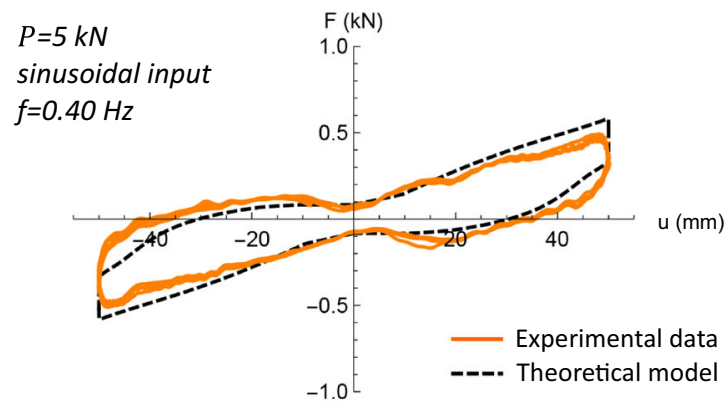

E

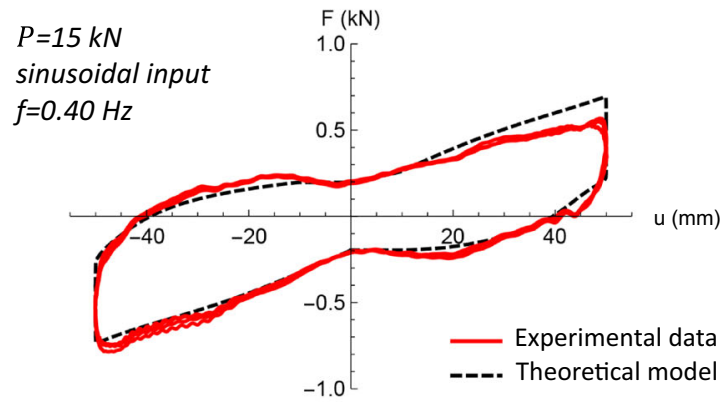

G

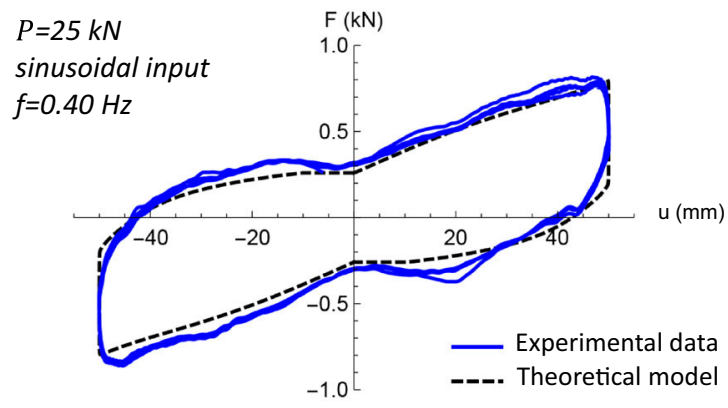

Fig. 5 Shear force $F$ versus lateral displacement $u$ response of the SSI prototype \#2. a Images of the tendons reinforced with M3 bolts at the extremities. b Components of the adopted mechanical model (sinusoidal loading with $P=25 \mathrm{kN}$ ). c-h) $F-u$ curves for $P=5 \mathrm{kN}(\mathbf{C}, \mathbf{D}) ; P=15 \mathrm{kN}(\mathrm{E}-\mathrm{F})$; and $P=25 \mathrm{kN}(\mathbf{g}-\mathbf{h})$.
B
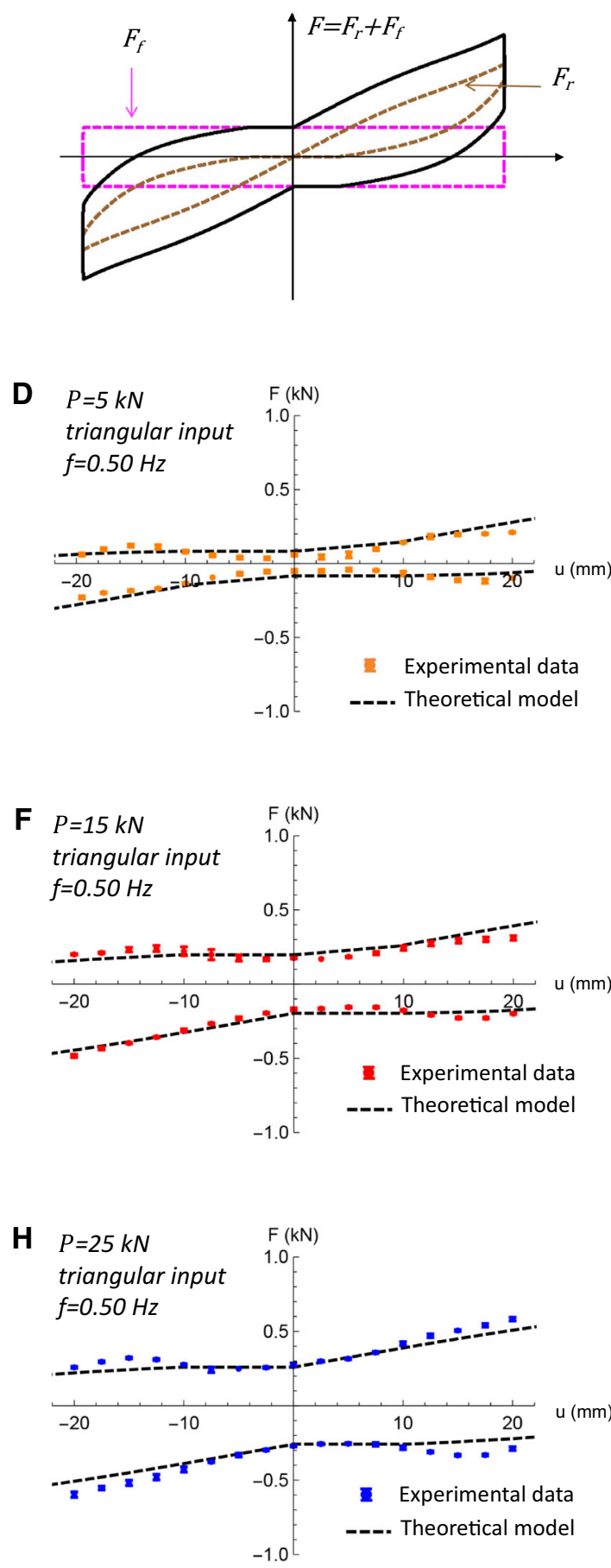

The displacement window of triangular loading tests has been restricted to $85 \%$ of the maximum value to exclude disturbance effects related to load reversal. Video recordings of selected tests are given in Movies S8-S10 
$\mathrm{kN})$, which are close to zero on the scale range of the horizontal actuator $(0-100 \mathrm{kN})$, and the experimentally measured variability of the vertical load $P$. Passing to examine the sinusoidal test on sample \#2 under $P=5$ $\mathrm{kN}$, we note that the loading branches of the experimental $F-u$ response exhibit low-slope segments in proximity to the points with $u=0$ (see Fig. 5c). Such portions of the $F-u$ curve are caused by the fact the unconditioned tendons are initially 'slack' due to the residual strains accumulated in the training cycle (see also Movie S8). In the tests with $P=15 \mathrm{kN}$, the initial slope of the $F-u$ curve (near $u=0$ ) is smaller for $u>0$ than for $u<0$, thanks to the insertion of two preconditioned tendons working for $u<0$ and only one working for $u>0$ (Fig. 5e and Movie S9). Finally, for $P=25 \mathrm{kN}$ all the loading branches of the $F-u$ curve exhibit considerably high slope near $u=0$, due to the fact that all the tendons were pre-conditioned in correspondence to such a value of $P$ (Fig. 5g and Movie S10). As a result, the overall matching between theoretical predictions and experimental observations of the force-displacement response is appreciably more accurate for $P=25 \mathrm{kN}$ than for the previous cases (cf. Fig. 5c,e,g).

Let us now compare the results of sinusoidal tests, affected by material- and friction-dependent nonlinearities (due to the time-variation of the sliding-velocity of the central post), with those of triangular input tests, where the sliding velocity is constant (see Figs. 4, 5). A comparative analysis of the response laws given in Figs. 4 and 5 reveals that the nonlinearity of the $F-u$ curve of prototype \#2 is essentially due to the nonlinear behavior of the tendons and pre-conditioning effects. The hysteretic response of the tendons determines the different shapes of the loading and unloading branches shown in Fig. 5d,f,h [43-45]. A featured video of the deformation of the tendons of prototype \#2 under testing $(P=25 \mathrm{kN})$ is given in Movie $\mathrm{S} 4$, while video recordings of the sinusoidal tests on prototypes \#1 and \#2 are provided in Movies S5-S10. Referring to the theoretical model for prototype \#2, we record $\xi_{\text {eff }}=$ $17.05 \%, 24.72 \%, 27.84 \%$ for $P=5 \mathrm{kN}, 15 \mathrm{kN}$ and 25 $\mathrm{kN}$, respectively. Similarly, we estimate $T_{\text {eff }}$ of prototype \#2, respectively, equal to $1.32 \mathrm{~s}, 2.09 \mathrm{~s}$ and $2.51 \mathrm{~s}$ for $P=5 \mathrm{kN}, 15 \mathrm{kN}$ and $25 \mathrm{kN}$. For $P=25 \mathrm{kN}$, the employed mechanical model estimates $T_{e f f}=1.94 \mathrm{~s}$ $\left(\xi_{\text {eff }}=20.87 \%\right)$ when setting the cross section area of the tendons $A_{t}$ to twice the value $A_{t_{0}}$ corresponding to prototype \#2 (cf. Fig. 3e).

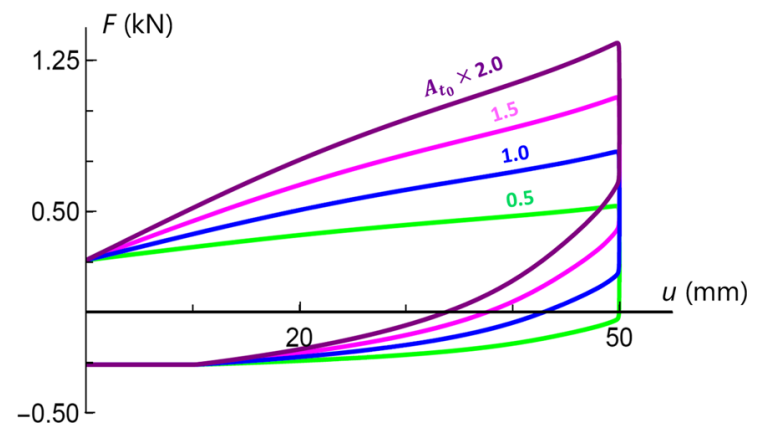

Fig. 6 Force-displacement responses of prototype \#2 for variable sizes of the tendons $(P=25 \mathrm{kN})$

\section{Scaling procedure}

We will now extrapolate the results obtained in the previous section to SSIs of different sizes and loaddisplacement capacities. It is easy to recognize that the load carrying capacity of the SSI prototypes can be adequately increased by playing with the diameters of the central post, central cap, central cushion and slider: a load carrying capacity of $250 \mathrm{kN}$, e.g., requires the adoption of a central post with a $\approx 41-\mathrm{mm}$ diameter, and a PTFE slider with a $\approx 95-\mathrm{mm}$ diameter, when using the same materials of prototypes \#1 and \#2 [49]. Alternatively, one can employ an array configuration (Fig. S2, B) to distribute the vertical load among smaller-size multiple posts, as we have already noticed. For what concerns the displacement capacity, it is possible to reach $d=500 \mathrm{~mm}$ using a one-layer system with limbs' length $a \approx 710 \mathrm{~mm}$ or a two-layer system with $a \approx 355 \mathrm{~mm}$.

The scaling law of the tendons can be obtained via the following formula inspired by international standards for seismic isolators [50]:

$F_{r_{d}}(P)=\chi P$

where $F_{r_{d}}$ denotes the design value of the restoring force to be carried out by the tendons, and $\chi$ is a dimensionless parameter. The 2001 California Building code [51] prescribes a minimum value of the post-elastic stiffness of a nonlinear isolation system such that the corresponding restoring force can be computed through the above formula with $\chi \geq 0.05$. The AASHTO code for seismic isolation [52] instead recommends $\chi \geq 0.025$ (in association with a suitable limitation of the fundamental period of the device, see [50] for further details). It is worth noting that the maximum value of the engineering strain that can be suffered by 
the tendons, in correspondence to the locking configuration $\left(\epsilon_{\text {lock }}\right)$, is independent of the limbs' length, which implies that such a quantity is also independent of the displacement capacity $d$, for a given value of $\beta$. Referring, e.g., to the case with $a_{1}=a_{2}=a$ and $\beta=0$, we observe that $\epsilon_{\text {lock }}$ is equal to $\sqrt{2}-1$, independent of the value of $a$ (see the Mathematica ${ }^{\circledR}$ code given in Supplementary Materials). $\epsilon_{\text {lock }}$ increases for $\beta<0$, reaching $\approx 0.55$ for $a_{1}=a_{2}=a$ and $\beta=-10$ deg.

We are now in a position to generalize Eqs. (5-6) to an arbitrary SSI. We estimate $\xi_{\text {eff }}$ and $T_{\text {eff }}$ of such a device through:

$\xi_{e f f}=\frac{2 \lambda \mu_{e f f}}{\pi\left(\mu_{e f f}+\chi\right)}$,

$$
T_{e f f}=2 \pi \sqrt{\frac{d}{\left(\mu_{e f f}+\chi\right) g}}
$$

where $\lambda$ is a scalar parameter defined by the ratio between the $E D C$ of a SSI equipped with tendons and that of the same device without the tendons. It is seen that the design variables $T_{\text {eff }}$ and $\xi_{\text {eff }}$ depend on the geometry of the device, the current value of $\mu_{e f f}$, the ratio $\chi$ between the design value of the recentering force of the tendons and the maximum vertical load, and the dimensionless parameter $\lambda$, which characterizes the energy dissipation capacity of the tendons (we recorded $\lambda$ variable between 1.87 , for $P=5 \mathrm{kN}$, and 1.34 , for $P=25 \mathrm{kN}$, during the tests run on prototype \#2).

The dynamic properties of the SSI can be effectively adjusted for the particular application at hand. Setting $\mu_{e f f}=2.0 \%$, we estimate that $T_{e f f}$ varies from $1.66 \mathrm{~s}\left(\xi_{\text {eff }}=11.57 \%\right)$ to $2.96 \mathrm{~s}\left(\xi_{\text {eff }}=27.68 \%\right)$ when one assumes in the design phase $(d= \pm 150$ $\mathrm{mm}, \chi=0.20, \lambda=2.0)$ and $(d= \pm 250 \mathrm{~mm}$, $\chi=0.095, \lambda=2.5$ ), respectively. The friction coefficient can be tuned by playing with the vertical load and the size and materials of the slider and the resting plate, while the tendons' energy dissipation properties can be adjusted through their preliminary training, in addition to an optimized design of the geometry and materials. The insertion of special dissipative elements at the mid-span of the tendons and the use of recycled materials [30] can also be considered.

\section{Concluding remarks}

We conclude that the biomimetic isolators analyzed in this study help us to forge a novel path to seismic isolation. Such devices with an anthropomorphic character are classified as highly tunable seismic isolators that can be manufactured with customized properties using optimal geometries and sustainable materials easily available around the world. Some key advantages enjoyed by these systems over their current state-ofthe-art counterparts are derived from the possibility to tune the displacement capacity acting only on the internal architecture of the unit cell; the uniaxial tension regime of the tendons, up to $40 \%-55 \%$ maximum axial strains, which is suitable for a class of materials much larger than the elastomeric products employed in rubber bearings [53]; and the possibility of creating periodic 'metaisolators' (Fig. S2, B). In addition, the SSI does not require heavy industry and is easily repaired by replacing the tendons after an extreme seismic event.

It is worth noting that the level of preliminary training of the tendons can be employed as a peculiar design variable of the SSI. We have observed that the reduced slope at the origin of the hysteretic loops of the device indicates a prevalent contribution of the frictional component to the base shear. By adjusting the amplitude of the pre-conditioning of the tendons one can suitably design the extension of this region of reduced reaction force. With such a design flexibility the building movement can be facilitated at the onset of the earthquake excitation, preventing the experience of significant levels of acceleration transferred to the structure, while providing higher stiffness for larger displacements.

The design and testing of architectured seismic isolators that feature an independently tunable antiseismic performance against horizontal and vertical ground motions will be addressed in future work, employing, e.g., linkages that mimic the knee articulation of the human leg in the vertical plane. Such a nonconventional performance is highly desirable in the case of high-risk industrial installations and power plants [5].

Acknowledgements The authors gratefully acknowledge the great support received by Filipe Santos (Universidade NOVA de Lisboa, Portugal) for what concerns the design and 3D printing of the demonstrative isolator prototypes, and the precious assistance received by Giuseppe Rocchetta (University of Salerno) in the development of numerical simulations. The technical support received by Maria Gabriella Castellano and Samuele Infanti (FIP 
MEC srl, Selvazzano Dentro, Padova, Italy) during the design and execution of laboratory tests is also gratefully acknowledged.

Author contributions G.W.M. formulated the initial concept of the isolator without the tendon elements. F.F. added the tendon members to the isolator concept and led the writing of the manuscript. G.B. included friction effects and designed and directed the experiments together with F.F. N.S. led the computeraided design and the manufacturing of the final isolator prototypes. A.A. was in charge of the mechanical modeling of the experimental results. All authors analyzed the results of the experimental tests and reviewed the manuscript.

Funding This study was funded by Italian Ministry of University and Research PRIN 2017 Grant 2017J4EAYB and US National Science Foundation Grant DMS-2107926.

Data Availability Statement The Mathematica ${ }^{\circledR}$ code employed for the prediction of the kinematics of the biomimetic isolator is available as an Appendix to Supplementary Text. The stereolithography (STL) files of all the unit cell parts of the demonstrative prototypes can be provided by the corresponding author upon request.

\section{Declarations}

Conflict of interests The authors declare that they have no conflict of interest.

Open Access This article is licensed under a Creative Commons Attribution 4.0 International License, which permits use, sharing, adaptation, distribution and reproduction in any medium or format, as long as you give appropriate credit to the original author(s) and the source, provide a link to the Creative Commons licence, and indicate if changes were made. The images or other third party material in this article are included in the article's Creative Commons licence, unless indicated otherwise in a credit line to the material. If material is not included in the article's Creative Commons licence and your intended use is not permitted by statutory regulation or exceeds the permitted use, you will need to obtain permission directly from the copyright holder. To view a copy of this licence, visit http://creativecommons.org/licenses/ by/4.0/.

\section{References}

1. Naeim, F., Kelly, J.M.: Design of Seismic Isolated Structures: From Theory to Practice. Wiley, New York (1999)

2. Clemente, P., Martelli, A.: Seismically isolated buildings in Italy: state-of-the-art review and applications. Soil Dyn. Earth. Eng. 119, 471-487 (2019). https://doi.org/10.1016/j. soildyn.2017.12.029

3. De Luca, A., Guidi, L.G.: State of the art in the worldwide evolution of base isolation design. Soil Dyn. Earthq. Eng. (2019). https://doi.org/10.1016/j.soildyn.2019.105722

4. Morales, E., Filiatrault, A., Aref, A.: Seismic floor isolation using recycled tires for essential buildings in develop- ing countries. B Earthq. Eng. 16, 6299-6333 (2018). https:// doi.org/10.1007/s10518-018-0416-7

5. Najafijozani, M., Becker, T.C., Konstantinidis, D.: Evaluating adaptive vertical seismic isolation for equipment in nuclear power plants. Nucl. Eng. Des. 358, 110399 (2020). https://doi.org/10.1016/j.nucengdes.2019.110399

6. Venanzi, I., Ierimonti, L., Materazzi, A.L.: Active base isolation of museum artifacts under seismic excitation. J. Earthq. Eng. 24, 506-527 (2020). https://doi.org/10.1080/ 13632469.2018.1453410

7. Kelly, J.M.: Earthquake-Resistant Design with Rubber. Springer, London (1993)

8. Lomiento, G., Bonessio, N., Benzoni, G.: Friction model for sliding bearings under seismic excitation. J. Earthq. Eng. 17, 1162-1191 (2013). https://doi.org/10.1080/13632469. 2013.814611

9. Rathje, E.M., Abrahamson, N.A., Bray, J.D.: Simplified frequency content estimates of earthquake ground motions. J. Geotech. Geoenviron. 124, 150-158 (1998). https://doi.org/ 10.1061/(ASCE)1090-0241(1998)124:2(150)

10. EN 15129. Anti-seismic devices. European committee for standardization (2009)

11. Hamaguchi, H., Aizawa, S., Samejima, Y., Kikuchi, T., Suzuki, S., Yoshizawa, T.: A study of aging effect on a rubber bearing after about twenty years in use. AIJ J. Technol. Des. 15(30), 393-398 (2009). https://doi.org/10.3130/aijt. 15.393

12. Liu, W., Ren, Y., He, W., Feng, D.: Aging and creep properties of LRB isolators used in building. World Inf. Earthq. Eng. 28(4), 131-136 (2012)

13. Calabrese, A., Losanno, D., Barjani, A., Spizzuoco, M., Strano, S.: Effects of the long-term aging of glass-fiber reinforced bearings (FRBs) on the seismic response of a baseisolated residential building. Eng. Struct. (2020). https://doi. org/10.1016/j.engstruct.2020.110735

14. Higashino, M., Hamaguchi, H.I., Minewaki, S., Aizawa, S.: Basic characteristics and durability of low-friction sliding bearings for base isolation. J. Earthq. Eng. Seism. 4, 95105 (2003)

15. Nakamura, Y., Okada, K.: Review on seismic isolation and response control methods of buildings in japan. Geoenviron Disasters (2019). https://doi.org/10. 1186/s40677-019-0123-y

16. Sierra, I.E.M., Losanno, D., Strano, S., Marulanda, J., Thomson, P.: Development and experimental behavior of HDR seismic isolators for low-rise residential buildings. Eng. Struct. 183, 894-906 (2019). https://doi.org/10.1016/ j.engstruct.2019.01.037

17. Spizzuoco, M., Calabrese, A., Serino, G.: Innovative lowcost recycled rubber-fiber reinforced isolator: experimental tests and finite element analyses. Eng. Struct. 76, 99-111 (2014). https://doi.org/10.1016/j.engstruct.2014.07.001

18. Tsiavos, A., Alexander, N.A., Diambra, A., Ibraim, E., Vardanega, P.J., Gonzalez-Buelga, A., Sextos, A.: A sandrubber deformable granular layer as a low-cost seismic isolation strategy in developing countries: experimental investigation. Soil Dyn. Earthq. Eng. 125, 105731 (2019). https:// doi.org/10.1016/j.soildyn.2019.105731

19. Losanno, D., Palumbo, F., Calabrese, A., Barrasso, T., Vaiana, N.: Preliminary investigation of aging effects on recycled rubber fiber reinforced bearings (RR-FRBs). 
J. Earthq. Eng. (2021). https://doi.org/10.1080/13632469. 2021.1871683

20. Barthelat, F.: Architectured materials in engineering and biology: fabrication, structure, mechanics and performance. Int. Mater. Rev. 60(8), 413-430 (2015). https://doi.org/10. 1179/1743280415Y.0000000008

21. Kadic, M., Milton, G.W., van Hecke, M., Wegener, M.: 3D metamaterials. Nat. Rev. Phys. 1, 198-210 (2019). https:// doi.org/10.1038/s42254-018-0018-y

22. Rafsanjani, A., Bertoldi, K., Studart, A.R.: Programming soft robots with flexible mechanical metamaterials. Sci. Robot. 29, eaav7874 (2019). https://doi.org/10.1126/ scirobotics.aav7874

23. Kotikian, A., McMahan, C., Davidson, E.C., Muhammad, J.M., Weeks, R.D., Daraio, C., Lewis, J.A.: Untethered soft robotic matter with passive control of shape morphing and propulsion. Sci. Robot. 4(33), eaax7044 (2019). https://doi. org/10.1126/scirobotics.aax7044

24. Milton, G.W.: New metamaterials with macroscopic behavior outside that of continuum elastodynamics. New J. Phys. (2007). https://doi.org/10.1088/1367-2630/9/10/359

25. Cummer, S.A., Christensen, J., Alu, A.: Controlling sound with acoustic metamaterials. Nat. Rev. Mater. 1, 16001 (2016). https://doi.org/10.1038/natrevmats.2016.1

26. Lepidi, M., Bacigalupo, A.: Wave propagation properties of one-dimensional acoustic metamaterials with nonlinear diatomic microstructure. Nonlinear Dyn. 98, 2711-2735 (2019). https://doi.org/10.1007/s11071-019-05032-3

27. Bukhari, M., Barry, O.: Spectro-spatial analyses of a nonlinear metamaterial with multiple nonlinear local resonators. Nonlinear Dyn. 99, 1539-1560 (2020). https://doi.org/10. 1007/s11071-019-05373-z

28. Frenzel, T., Kadic, M., Wegener, M.: Three-dimensional mechanical metamaterials with a twist. Science 358(6366), 1072-1074 (2017). https://doi.org/10.1126/science. aao4640

29. Falahati, M., Ahmadvand, P., Safaee, S., Chang, Y.-C., Lyu, Z., Chen, R., Li, L., Lin, Y.: Smart polymers and nanocomposites for 3D and 4D printing. Mater. Today 40, 215-245 (2020). https://doi.org/10.1016/j.mattod.2020.06.001

30. Cruz Sanchez, F.A., Boudaoud, H., Camargo, M., Pearce, J.M.: Plastic recycling in additive manufacturing: A systematic literature review and opportunities for the circular economy. J Clean. Prod. 264, 121602 (2020). https://doi. org/10.1016/j.jclepro.2020.121602

31. Colombi, A., Colquitt, D., Roux, P., Guenneau, S., Craster, R.V.: A seismic metamaterial: the resonant metawedge. Sci. Rep. 6, 27717 (2016). https://doi.org/10.1038/srep27717

32. Palermo, A., Krödel, S., Marzani, A., Daraio, C.: Engineered metabarrier as shield from seismic surface waves. Sci. Rep. (2016). https://doi.org/10.1038/srep39356

33. Du, Q., Zeng, Y., Huang, G., Yang, H.: Elastic metamaterialbased seismic shield for both lamb and surface waves. AIP Adv. 7, 075015 (2017). https://doi.org/10.1063/1.4996716

34. Brûlé, S., Ungureanu, B., Achaoui, Y., Diatta, A., Aznavourian, R., Antonakakis, T., Craster, R., Enoch, S., Guenneau, S.: Metamaterial-like transformed urbanism. Innov. Infrastruct. Solut. 2(1), 20 (2017)

35. Brûlé, S., Enoch, S., Guenneau, S.: Emergence of seismic metamaterials: current state and future perspectives. Phys. Lett. A 384(1), 1-11 (2020)
36. Krasnok, A., Vellucci, S., Miniaci, M., Kherraz, N., Croënne, C., Mazzotti, M., Morvaridi, M., Gliozzi, A.S., Onorato, M., Bosia, F., Pugno, N.M.: Hierarchical largescale elastic metamaterials for passive seismic wave mitigation. EPJ Appl. Metamater. (2021). https://doi.org/10.1051/ epjam/2021009

37. Fraternali, F., Amendola, A.: Mechanical modeling of innovative metamaterials alternating pentamode lattices and confinement plates. J. Mech. Phys. Solids 99, 259-271 (2017). https://doi.org/10.1016/j.jmps.2016.11.010

38. Clayton, M., Philo, R.: Leonardo Da Vinci: anatomist. Royal Collection Trust (2012). ISBN $978 \quad 1909741$ 034 (https://www.rct.uk/collection/themes/publications/ leonardo-da-vinci-anatomisthttps://www.rct.uk)

39. Ahlborn, B.K., Blake, R.W., Megill, W.M.: Frequency tuning in animal locomotion. Zoology 109, 43-53 (2006). https://doi.org/10.1016/j.zool.2005.11.001

40. Alexander, R.M.: Mechanics of skeleton and tendons. In: Prakash Y.S. (ed.) Comprehensive Physiology (2011)

41. Feng, X., Jing, X., Xu, Z., Guo, Y.: Bio-inspired antivibration with nonlinear inertia coupling. Mech. Syst. Signal. Pr. 124, 562-595 (2019). https://doi.org/10.1016/j. ymssp.2019.02.001

42. Chen, J., Liao, W.H.: Design and control of a magnetorheological actuator for leg exoskeleton. Proc. IEEE Int. Conf. ROBIO, Sanya, China 1388-1393 (2007). https:// ieeexplore.ieee.org/document/4522367https://doi.org/10. 1109/ROBIO.2007.4522367

43. Dorfmann, A., Ogden, R.W.: A pseudo-elastic model for loading, partial unloading and reloading of particlereinforced rubber. Int. J. Solids Struct. 40, 2699-2714 (2003). https://doi.org/10.1016/S0020-7683(03)00089-1

44. Dorfmann, A., Ogden, R.W.: A constitutive model for the Mullins effect with permanent set in particle-reinforced rubber. Int. J. Solids Struct. 42, 2699-2714 (2003). https://doi. org/10.1016/j.ijsolstr.2003.11.014

45. Qi, H.J., Boyce, M.C.: Stress-strain behavior of thermoplastic polyurethanes. Mech. Mater. 37(8), 817-839 (2005). https://doi.org/10.1016/j.mechmat.2004.08.001

46. Chen, H., Trivedi, A.R., Siviour, C.R.: Application of linear viscoelastic continuum damage theory to the low and high strain rate response of thermoplastic polyurethane. Exp. Mech. 60(7), 925-936 (2020)

47. Mohotti, D., Ali, M., Ngo, T., Lu, J., Mendis, P.: Strain rate dependent constitutive model for predicting the material behaviour of polyurea under high strain rate tensile loading. Mater. Des. 53, 830-837 (2014). https://doi.org/10.1016/j. matdes.2013.07.020

48. Eurocode 8: Design of structures for earthquake resistance. EN1998-2. European Committee for Standardization, Bruxelles, Belgium (2005)

49. Beer, F.P., Johnston, E.R., DeWolf, J.T., Mazurek, D.F.: Mechanics of Materials, 9th edn. McGraw-Hill, New York (2020)

50. Cardone, D., Gesualdi, G., Brancato, P.: Restoring capability of friction pendulum seismic isolation systems. Bull. Earthq. Eng. 13, 2449-2480 (2015). https://doi.org/10. 1007/s10518-014-9719-5

51. CBSC: California Building Code. California Buildings Standards Commission, Sacramento (2001) 
52. AASHTO: Guide Specifications for Seismic Isolation Design. American Association of State Highways and Transportation Officials, Washington (2000)

53. Nishi, T., Suzuki, S., Aoki, M., Sawada, T., Fukuda, S.: International investigation of shear displacement capacity of various elastomeric seismic-protection isolators for buildings. J. Rubber Res. 22, 33-41 (2019). https://doi.org/10.1007/ s42464-019-00006-X
Publisher's Note Springer Nature remains neutral with regard to jurisdictional claims in published maps and institutional affiliations. 